\title{
Classes of an univalent integral operator
}

\author{
Camelia Bărbatu and Daniel Breaz
}

\begin{abstract}
In this paper we introduce a new general integral operator for analytic functions in the open unit disk $\mathbf{U}$ and we obtain sufficient conditions for univalence of this integral operator.
\end{abstract}

Mathematics Subject Classification (2010): 30C45.

Keywords: Integral operator, analytic and univalent functions, unit disk.

\section{Introduction}

Let $\mathcal{A}$ be the class of the functions $f$ which are analytic in the open unit disk $\mathbb{U}=\{z \in \mathbb{C}:|z|<1\}$ and $f(0)=f^{\prime}(0)-1=0$.

We denote by $\mathcal{S}$ the subclass of $\mathcal{A}$ consisting of functions $f \in \mathcal{A}$, which are univalent in $\mathbb{U}$.

We consider the integral operator

$$
\mathcal{T}_{n}(z)=\left\{\delta \int_{0}^{z} t^{\delta-1} \prod_{i=1}^{n}\left[\left(\frac{f_{i}(t)}{t}\right)^{\alpha_{i}-1} \cdot\left(g_{i}(t)^{\prime}\right)^{\beta_{i}} \cdot\left(\frac{h_{i}(t)}{k_{i}(t)}\right)^{\gamma_{i}} \cdot\left(\frac{\left.h_{i}{ }^{\prime}(t)\right)}{k_{i}{ }^{\prime}(t)}\right)^{\delta_{i}}\right] \mathrm{dt}\right\}^{\frac{1}{\delta}}
$$

for $f_{i}, g_{i}, h_{i}, k_{i} \in \mathcal{A}$ and the complex numbers $\delta, \alpha_{i}, \beta_{i}, \gamma_{i}, \delta_{i}$, with $\delta \neq 0, i=\overline{1, n}$, $n \in \mathbb{N} \backslash\{0\}$.

Remark 1.1. The integral operator $\mathcal{T}_{n}$ defined by (1.1), is a general integral operator of Pfaltzgraff, Kim-Merkes and Ovesea types which extends also the other operators as follows:

i) For $n=1, \delta=1, \alpha_{1}-1=\alpha_{1}$ and $\beta_{1}=\gamma_{1}=\delta_{1}=0$ we obtain the integral operator which was studied by Kim-Merkes [7].

$$
\mathcal{F}_{\alpha}(z)=\int_{0}^{z}\left(\frac{f(t)}{t}\right)^{\alpha} \mathrm{dt}
$$


ii) For $n=1, \delta=1$ and $\alpha_{1}-1=\gamma_{1}=\delta_{1}=0$ we obtain the integral operator which was studied by Pfaltzgraff [18].

$$
\mathcal{G}_{\alpha}(z)=\int_{0}^{z}\left(f^{\prime}(t)\right)^{\alpha} \mathrm{dt},
$$

iii) For $\alpha_{i}-1=\alpha_{i}$ and $\beta_{i}=\gamma_{i}=\delta_{i}=0$ we obtain the integral operator which was defined and studied by D. Breaz and N. Breaz [2].

$$
\mathcal{D}_{n}(z)=\left[\delta \int_{0}^{z} t^{\delta-1} \prod_{i=1}^{n}\left(\frac{f_{i}(t)}{t}\right)^{\alpha_{i}} \mathrm{dt}\right]^{\frac{1}{\delta}}
$$

this integral operator is a generalization of the integral operator introduced by Pascu and Pescar [12].

iv) For $\alpha_{i}-1=\gamma_{i}=\delta_{i}=0$ we obtain the integral operator which was defined and studied by D. Breaz, Owa and N. Breaz [4]

$$
\mathcal{I}_{n}(z)=\left[\delta \int_{0}^{z} t^{\delta-1} \prod_{i=1}^{n}\left[f_{i}^{\prime}(t)\right]^{\alpha_{i}} \mathrm{dt}\right]^{\frac{1}{\delta}}
$$

this integral operator is a generalization of the integral operator introduced by Pescar and Owa in [17].

v) For $\alpha_{i}-1=\alpha_{i}$ and $\gamma_{i}=\delta_{i}=0$ we obtain the integral operator which was defined and studied by Frasin [5]

$$
\mathcal{F}_{n}(z)=\left[\delta \int_{0}^{z} t^{\delta-1} \prod_{i=1}^{n}\left(\frac{f_{i}(t)}{t}\right)^{\alpha_{i}}\left(f_{i}{ }^{\prime}(t)\right)^{\beta_{i}} \mathrm{dt}\right]^{\frac{1}{\delta}},
$$

this integral operator is a generalization of the integral operator introduced by Ovesea in $[9]$.

vi) For $\alpha_{i}-1=\beta_{i}=0$ we obtain the integral operator which was defined and studied by Pescar [13].

$$
\mathcal{I}_{n}(z)=\left[\delta \int_{0}^{z} t^{\delta-1} \prod_{i=1}^{n}\left(\frac{f_{i}(t)}{g_{i}(t)}\right)^{\gamma_{i}}\left(\frac{f_{i}^{\prime}(t)}{g_{i}^{\prime}(t)}\right)^{\delta_{i}} \mathrm{dt}\right]^{\frac{1}{\delta}},
$$

Thus, the integral operator $\mathcal{T}_{n}$, introduced here by the formula (1.1), can be considered as an extension and a generalization of these operators above mentioned.

We need the following lemmas.

Lemma 1.2. [11] Let $\gamma, \delta$ be complex numbers, Re $\gamma>0$ and $f \in \mathcal{A}$. If

$$
\frac{1-|z|^{2 \operatorname{Re} \gamma}}{\operatorname{Re\gamma }}\left|\frac{\left.z f^{\prime \prime}(z)\right)}{f^{\prime}(z)}\right| \leq 1
$$

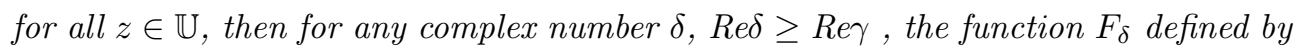

$$
F_{\delta}(z)=\left(\delta \int_{0}^{z} t^{\delta-1} f^{\prime}(t) d t\right)^{\frac{1}{\delta}}
$$


is regular and univalent in $\mathbb{U}$.

Lemma 1.3. [14] Let $\delta$ be complex number, Re $\delta>0$ and c a complex number, $|c| \leq 1$, $c \neq-1$, and $f \in \mathcal{A}, f(z)=z+a_{2} z^{2}+\ldots$. If

$$
\left.|c| z\right|^{2 \delta}+\left(1-|z|^{2 \delta}\right) \frac{\left.z f^{\prime \prime}(z)\right)}{\delta f^{\prime}(z)} \mid \leq 1,
$$

for all $z \in \mathbb{U}$, then the function $F_{\delta}$ defined by

$$
F_{\delta}(z)=\left(\delta \int_{0}^{z} t^{\delta-1} f^{\prime}(t) d t\right)^{\frac{1}{\delta}}
$$

is regular and univalent in $\mathbb{U}$.

Lemma 1.4. [8] Let $f$ be the function regular in the disk $\mathbb{U}_{R}=\{z \in \mathbb{C}:|z|<R\}$ with $|f(z)|<M$, M fixed. If $f(z)$ has in $z=0$ one zero with multiply $\geq m$, then

$$
|f(z)| \leq \frac{M}{R^{m}} z^{m}
$$

the equality for $z \neq 0$ can hold only if

$$
f(z)=e^{i \theta} \frac{M}{R^{m}} z^{m}
$$

where $\theta$ is constant.

\section{Main results}

Theorem 2.1. Let $\gamma, \delta, \alpha_{i}, \beta_{i}, \gamma_{i}, \delta_{i}$ be complex numbers, $c=\operatorname{Re} \gamma>0, M_{i}, N_{i}, P_{i}, Q_{i}$, $R_{i}, S_{i}$ real positive numbers and $f_{i}, g_{i}, h_{i}, k_{i} \in \mathcal{A}$,

$$
\begin{aligned}
& f_{i}(z)=z+a_{2 i} z^{2}+a_{3 i} z^{3}+\ldots, \\
& g_{i}(z)=z+b_{2 i} z^{2}+b_{3 i} z^{3}+\ldots, \\
& h_{i}(z)=z+c_{2 i} z^{2}+c_{3 i} z^{3}+\ldots, \\
& k_{i}(z)=z+d_{2 i} z^{2}+d_{3 i} z^{3}+\ldots, \quad i=\overline{1, n} .
\end{aligned}
$$

If

$$
\begin{gathered}
\left|\frac{z f_{i}^{\prime}(z)}{f_{i}(z)}-1\right| \leq M_{i}, \quad\left|\frac{z g_{i}^{\prime}(z)}{g_{i}(z)}-1\right| \leq N_{i}, \quad\left|\frac{z h_{i}^{\prime}(z)}{h_{i}(z)}-1\right| \leq P_{i}, \\
\left|\frac{z k_{i}^{\prime}(z)}{k_{i}(z)}-1\right| \leq Q_{i}, \quad\left|\frac{z h_{i}^{\prime \prime}(z)}{h_{i}^{\prime}(z)}\right| \leq R_{i}, \quad\left|\frac{z k_{i}^{\prime \prime}(z)}{k_{i}^{\prime}(z)}\right| \leq S_{i},
\end{gathered}
$$

for all $z \in \mathbb{U}, i=\overline{1, n}$ and

$$
\sum_{i=1}^{n}\left[\left|\alpha_{i}-1\right| M_{i}+\left|\beta_{i}\right| N_{i}+\left|\gamma_{i}\right|\left(P_{i}+Q_{i}\right)+\left|\delta_{i}\right|\left(R_{i}+S_{i}\right)\right] \leq \frac{(2 c+1)^{\frac{2 c+1}{2 c}}}{2},
$$

then, for all $\delta$ complex numbers, Re $\delta \geq \operatorname{Re} \gamma$, the integral operator $\mathcal{T}_{n}$, given by (1.1) is in the class $\mathcal{S}$. 
Proof. Let us define the function

$$
H_{n}(z)=\int_{0}^{z} \prod_{i=1}^{n}\left[\left(\frac{f_{i}(t)}{t}\right)^{\alpha_{i}-1} \cdot\left(g_{i}(t)^{\prime}\right)^{\beta_{i}} \cdot\left(\frac{h_{i}(t)}{k_{i}(t)}\right)^{\gamma_{i}} \cdot\left(\frac{h_{i}{ }^{\prime}(t)}{k_{i}{ }^{\prime}(t)}\right)^{\delta_{i}}\right] \mathrm{dt},
$$

for $f_{i}, g_{i}, h_{i}, k_{i} \in \mathcal{A}, i=\overline{1, n}$.

The function $H_{n}$ is regular in $\mathbb{U}$ and satisfy the following usual normalization conditions $H_{n}(0)=H_{n}^{\prime}(0)-1=0$.

Now

$$
H_{n}{ }^{\prime}(z)=\prod_{i=1}^{n}\left[\left(\frac{f_{i}(t)}{t}\right)^{\alpha_{i}-1} \cdot\left(g_{i}(t)^{\prime}\right)^{\beta_{i}} \cdot\left(\frac{h_{i}(z)}{k_{i}(z)}\right)^{\gamma_{i}} \cdot\left(\frac{h_{i}^{\prime}(z)}{k_{i}^{\prime}(z)}\right)^{\delta_{i}}\right] .
$$

We have

$$
\begin{gathered}
\frac{z H_{n}^{\prime \prime}(z)}{H_{n}^{\prime}(z)}=\sum_{i=1}^{n}\left[\left(\alpha_{i}-1\right)\left(\frac{z f_{i}^{\prime}(z)}{f_{i}(z)}-1\right)+\beta_{i} \frac{z g_{i}^{\prime \prime}(z)}{g_{i}^{\prime}(z)}\right] \\
+\sum_{i=1}^{n}\left[\gamma_{i}\left(\frac{z h_{i}^{\prime}(z)}{h_{i}(z)}-\frac{z k_{i}^{\prime}(z)}{k_{i}(z)}\right)+\delta_{i}\left(\frac{z h_{i}^{\prime \prime}(z)}{h_{i}^{\prime}(z)}-\frac{z k_{i}^{\prime \prime}(z)}{k_{i}^{\prime}(z)}\right)\right],
\end{gathered}
$$

for all $z \in \mathbb{U}$.

Thus, we have

for all $z \in \mathbb{U}$.

$$
\begin{gathered}
\frac{1-|z|^{2 c}}{c}\left|\frac{z H_{n}^{\prime \prime}(z)}{H_{n}^{\prime}(z)}\right|=\frac{1-|z|^{2 c}}{c} \sum_{i=1}^{n}\left[\left(\alpha_{i}-1\right)\left(\frac{z f_{i}^{\prime}(z)}{f_{i}(z)}-1\right)+\beta_{i} \frac{z g_{i}^{\prime \prime}(z)}{g_{i}^{\prime}(z)}\right] \\
+\frac{1-|z|^{2 c}}{c} \sum_{i=1}^{n}\left[\gamma_{i}\left(\frac{z h_{i}^{\prime}(z)}{h_{i}(z)}-\frac{z k_{i}^{\prime}(z)}{k_{i}(z)}\right)+\delta_{i}\left(\frac{z h_{i}^{\prime \prime}(z)}{h_{i}^{\prime}(z)}-\frac{z k_{i}^{\prime \prime}(z)}{k_{i}^{\prime}(z)}\right)\right],
\end{gathered}
$$

Therefore

$$
\begin{aligned}
\frac{1-|z|^{2 c}}{c}\left|\frac{z H_{n}^{\prime \prime}(z)}{H_{n}^{\prime}(z)}\right| & \leq \frac{1-|z|^{2 c}}{c} \sum_{i=1}^{n}\left[\left|\alpha_{i}-1\right|\left|\frac{z f_{i}^{\prime}(z)}{f_{i}(z)}-1\right|+\left|\beta_{i}\right|\left|\frac{z g_{i}^{\prime \prime}(z)}{g_{i}^{\prime}(z)}\right|\right] \\
& +\frac{1-|z|^{2 c}}{c} \sum_{i=1}^{n}\left[\left|\gamma_{i}\right|\left(\left|\frac{z h_{i}^{\prime}(z)}{h_{i}(z)}-1\right|+\left|\frac{z k_{i}^{\prime}(z)}{k_{i}(z)}-1\right|\right)\right] \\
& +\frac{1-|z|^{2 c}}{c} \sum_{i=1}^{n}\left[\left|\delta_{i}\right|\left(\left|\frac{z h_{i}^{\prime \prime}(z)}{h_{i}^{\prime}(z)}\right|+\left|\frac{z k_{i}^{\prime \prime}(z)}{k_{i}^{\prime}(z)}\right|\right)\right]
\end{aligned}
$$

for all $z \in \mathbb{U}$.

By applying the General Schwarz Lemma (1.4) we obtain

$$
\begin{gathered}
\left|\frac{z f_{i}^{\prime}(z)}{f_{i}(z)}-1\right| \leq M_{i}|z|, \quad\left|\frac{z g_{i}^{\prime \prime}(z)}{g_{i}^{\prime}(z)}\right| \leq N_{i}|z|, \quad\left|\frac{z h_{i}^{\prime}(z)}{h_{i}(z)}-1\right| \leq P_{i}|z|, \\
\left|\frac{z k_{i}^{\prime}(z)}{k_{i}(z)}-1\right| \leq Q_{i}|z|, \quad\left|\frac{z h_{i}^{\prime \prime}(z)}{h_{i}^{\prime}(z)}\right| \leq R_{i}|z|, \quad\left|\frac{z K_{i}^{\prime \prime}(z)}{K_{i}^{\prime}(z)}\right| \leq S_{i}|z|,
\end{gathered}
$$

for all $z \in \mathbb{U}, i=\overline{1, n}$. 
Using these inequalities we have

$$
\begin{array}{r}
\frac{1-|z|^{2 c}}{c}\left|\frac{z H_{n}^{\prime \prime}(z)}{H_{n}^{\prime}(z)}\right| \\
\leq \frac{1-|z|^{2 c}}{c}|z| \sum_{i=1}^{n}\left[\left|\alpha_{i}-1\right| M_{i}+\left|\beta_{i}\right| N_{i}+\left|\gamma_{i}\right|\left(P_{i}+Q_{i}\right)+\left|\delta_{i}\right|\left(R_{i}+S_{i}\right)\right],
\end{array}
$$

for all $z \in \mathbb{U}$.

Since

$$
\max _{|z| \leq 1} \frac{\left(1-|z|^{2 c}\right)|z|}{c}=\frac{2}{(2 c+1)^{\frac{2 c+1}{2 c}}}
$$

from (2.2), we obtain

$$
\begin{gathered}
\frac{1-|z|^{2 c}}{c}\left|\frac{z H_{n}^{\prime \prime}(z)}{H_{n}^{\prime}(z)}\right| \\
\leq \frac{2}{(2 c+1)^{\frac{2 c+1}{2 c}}} \sum_{i=1}^{n}\left[\left|\alpha_{i}-1\right| M_{i}+\left|\beta_{i}\right| N_{i}+\left|\gamma_{i}\right|\left(P_{i}+Q_{i}\right)+\left|\delta_{i}\right|\left(R_{i}+S_{i}\right)\right],
\end{gathered}
$$

and hence, by (2.1) we have

$$
\frac{1-|z|^{2 c}}{c}\left|\frac{z H_{n}^{\prime \prime}(z)}{H_{n}^{\prime}(z)}\right| \leq \frac{2}{(2 c+1)^{\frac{2 c+1}{2 c}}} \cdot \frac{(2 c+1)^{\frac{2 c+1}{2 c}}}{2}=1,
$$

for all $z \in \mathbb{U}$.

So,

$$
\frac{1-|z|^{2 c}}{c}\left|\frac{z H_{n}^{\prime \prime}(z)}{H_{n}^{\prime}(z)}\right| \leq 1
$$

and using (2.3), by Lemma 1.2, it results that the integral operator $\mathcal{T}_{n}$, given by (1.1) is in the class $\mathcal{S}$.

If we consider $\delta=1$ in Theorem 2.1, obtain the next corollary:

Corollary 2.2. Let $\gamma, \alpha_{i}, \beta_{i}, \gamma_{i}, \delta_{i}$ be complex numbers, $0<$ Re $\gamma \leq 1, c=$ Re $\gamma$, $M_{i}, N_{i}, P_{i}, Q_{i}, R_{i}, S_{i}$ real positive numbers and $f_{i}, g_{i}, h_{i}, k_{i} \in \mathcal{A}$. If

$$
\begin{gathered}
\left|\frac{z f_{i}^{\prime}(z)}{f_{i}(z)}-1\right| \leq M_{i}, \quad\left|\frac{z g_{i}^{\prime \prime}(z)}{g_{i}^{\prime}(z)}\right| \leq N_{i}, \quad\left|\frac{z h_{i}^{\prime}(z)}{h_{i}(z)}-1\right| \leq P_{i}, \\
\left|\frac{z k_{i}^{\prime}(z)}{k_{i}(z)}-1\right| \leq Q_{i}, \quad\left|\frac{z h_{i}^{\prime \prime}(z)}{h_{i}^{\prime}(z)}\right| \leq R_{i}, \quad\left|\frac{z k_{i}^{\prime \prime}(z)}{k_{i}^{\prime}(z)}\right| \leq S_{i}, \\
\sum_{i=1}^{n}\left[\left|\alpha_{i}-1\right| M_{i}+\left|\beta_{i}\right| N_{i}+\left|\gamma_{i}\right|\left(P_{i}+Q_{i}\right)+\left|\delta_{i}\right|\left(R_{i}+S_{i}\right)\right] \leq \frac{(2 c+1)^{\frac{2 c+1}{2 c}}}{2},
\end{gathered}
$$

then the integral operator $\mathcal{F}_{n}$ defined by

$$
\mathcal{F}_{n}(z)=\int_{0}^{z} \prod_{i=1}^{n}\left[\left(\frac{f_{i}(t)}{t}\right)^{\alpha_{i}-1} \cdot\left(g_{i}(t)^{\prime}\right)^{\beta_{i}} \cdot\left(\frac{h_{i}(t)}{k_{i}(t)}\right)^{\gamma_{i}} \cdot\left(\frac{\left.h_{i}{ }^{\prime}(t)\right)}{k_{i}{ }^{\prime}(t)}\right)^{\delta_{i}}\right] d t
$$

is in the class $\mathcal{S}$. 
If we consider $\delta=1$ and $\delta_{1}=\delta_{2}=\ldots=\delta_{n}=0$ in Theorem 2.1, obtain the next corollary:

Corollary 2.3. Let $\gamma, \alpha_{i}, \beta_{i}, \gamma_{i}$ be complex numbers, $0<$ Re $\gamma \leq 1, c=$ Re $\gamma$, $M_{i}, N_{i}, P_{i}, Q_{i}$ real positive numbers and $f_{i}, g_{i}, h_{i}, k_{i} \in \mathcal{A}$. If

$$
\begin{gathered}
\left|\frac{z f_{i}^{\prime}(z)}{f_{i}(z)}-1\right| \leq M_{i}, \quad\left|\frac{z g_{i}^{\prime \prime}(z)}{g_{i}^{\prime}(z)}\right| \leq N_{i}, \\
\left|\frac{z h_{i}^{\prime}(z)}{h_{i}(z)}-1\right| \leq P_{i}, \quad\left|\frac{z k_{i}^{\prime}(z)}{k_{i}(z)}-1\right| \leq Q_{i},
\end{gathered}
$$

for all $z \in \mathbb{U}, i=\overline{1, n}$ and

$$
\sum_{i=1}^{n}\left[\left|\alpha_{i}-1\right| M_{i}+\left|\beta_{i}\right| N_{i}+\left|\gamma_{i}\right|\left(P_{i}+Q_{i}\right)\right] \leq \frac{(2 c+1)^{\frac{2 c+1}{2 c}}}{2},
$$

then the integral operator $\mathcal{S}_{n}$ defined by

$$
\mathcal{S}_{n}(z)=\int_{0}^{z} \prod_{i=1}^{n}\left[\left(\frac{f_{i}(t)}{t}\right)^{\alpha_{i}-1} \cdot\left(g_{i}(t)^{\prime}\right)^{\beta_{i}} \cdot\left(\frac{h_{i}(t)}{k_{i}(t)}\right)^{\gamma_{i}}\right] d t
$$

is in the class $\mathcal{S}$. corollary:

If we consider $\delta=1$ and $\beta_{1}=\beta_{2}=\ldots=\beta_{n}=0$ in Theorem 2.1, obtain the next

Corollary 2.4. Let $\gamma, \alpha_{i}, \gamma_{i}, \delta_{i}$ be complex numbers, $0<\operatorname{Re} \gamma \leq 1, c=$ Re $\gamma$, $M_{i}, P_{i}, Q_{i}, R_{i}, S_{i}$ real positive numbers and $f_{i}, h_{i}, k_{i} \in \mathcal{A}$. If

$$
\begin{gathered}
\left|\frac{z f_{i}^{\prime}(z)}{f_{i}(z)}-1\right| \leq M_{i}, \quad\left|\frac{z h_{i}^{\prime}(z)}{h_{i}(z)}-1\right| \leq P_{i}, \quad\left|\frac{z k_{i}^{\prime}(z)}{k_{i}(z)}-1\right| \leq Q_{i}, \\
\left|\frac{z h_{i}^{\prime \prime}(z)}{h_{i}^{\prime}(z)}\right| \leq R_{i}, \quad\left|\frac{z k_{i}^{\prime \prime}(z)}{k_{i}^{\prime}(z)}\right| \leq S_{i},
\end{gathered}
$$

for all $z \in \mathbb{U}, i=\overline{1, n}$ and

$$
\sum_{i=1}^{n}\left[\left|\alpha_{i}-1\right| M_{i}+\left|\gamma_{i}\right|\left(P_{i}+Q_{i}\right)+\left|\delta_{i}\right|\left(R_{i}+S_{i}\right)\right] \leq \frac{(2 c+1)^{\frac{2 c+1}{2 c}}}{2}
$$

then the integral operator $\mathcal{X}_{n}$ defined by

$$
\mathcal{X}_{n}(z)=\int_{0}^{z} \prod_{i=1}^{n}\left[\left(\frac{f_{i}(t)}{t}\right)^{\alpha_{i}-1} \cdot\left(\frac{h_{i}(t)}{k_{i}(t)}\right)^{\gamma_{i}} \cdot\left(\frac{\left.h_{i}{ }^{\prime}(t)\right)}{k_{i}{ }^{\prime}(t)}\right)^{\delta_{i}}\right] d t
$$

is in the class $\mathcal{S}$.

If we consider $\delta=1$ and $\alpha_{1}=\alpha_{2}=\ldots=\alpha_{n}=0$ in Theorem 2.1, obtain the next corollary: 
Corollary 2.5. Let $\gamma, \beta_{i}, \gamma_{i}, \delta_{i}$ be complex numbers, $0<\operatorname{Re} \gamma \leq 1, c=$ Re $\gamma$, $N_{i}, P_{i}, Q_{i}, R_{i}, S_{i}$ real positive numbers and $g_{i}, h_{i}, k_{i} \in \mathcal{A}$. If

for all $z \in \mathbb{U}, i=\overline{1, n}$ and

$$
\begin{gathered}
\left|\frac{z g_{i}^{\prime \prime}(z)}{g_{i}^{\prime}(z)}\right| \leq N_{i}, \quad\left|\frac{z h_{i}^{\prime}(z)}{h_{i}(z)}-1\right| \leq P_{i}, \quad\left|\frac{z k_{i}^{\prime}(z)}{k_{i}(z)}-1\right| \leq Q_{i}, \\
\left|\frac{z h_{i}^{\prime \prime}(z)}{h_{i}^{\prime}(z)}\right| \leq R_{i}, \quad\left|\frac{z k_{i}^{\prime \prime}(z)}{k_{i}^{\prime}(z)}\right| \leq S_{i},
\end{gathered}
$$

$$
\sum_{i=1}^{n}\left[\left|\beta_{i}\right| N_{i}+\left|\gamma_{i}\right|\left(P_{i}+Q_{i}\right)+\left|\delta_{i}\right|\left(R_{i}+S_{i}\right)\right] \leq \frac{(2 c+1)^{\frac{2 c+1}{2 c}}}{2}
$$

then the integral operator $\mathcal{D}_{n}$ defined by

$$
\mathcal{D}_{n}(z)=\int_{0}^{z} \prod_{i=1}^{n}\left[\left(g_{i}(t)^{\prime}\right)^{\beta_{i}} \cdot\left(\frac{h_{i}(t)}{k_{i}(t)}\right)^{\gamma_{i}} \cdot\left(\frac{\left.h_{i}{ }^{\prime}(t)\right)}{k_{i}{ }^{\prime}(t)}\right)^{\delta_{i}}\right] d t
$$

is in the class $\mathcal{S}$.

If we consider $\delta=1$ and $\gamma_{1}=\gamma_{2}=\ldots=\gamma_{n}=0$ in Theorem 2.1, obtain the next corollary:

Corollary 2.6. Let $\gamma, \alpha_{i}, \beta_{i}, \delta_{i}$ be complex numbers, $0<$ Re $\gamma \leq 1, c=$ Re $\gamma$, $M_{i}, N_{i}, R_{i}, S_{i}$ real positive numbers and $f_{i}, g_{i}, h_{i}, k_{i} \in \mathcal{A}$. If

for all $z \in \mathbb{U}, i=\overline{1, n}$ and

$$
\begin{gathered}
\left|\frac{z f_{i}^{\prime}(z)}{f_{i}(z)}-1\right| \leq M_{i}, \quad\left|\frac{z g_{i}^{\prime \prime}(z)}{g_{i}^{\prime}(z)}\right| \leq N_{i}, \\
\left|\frac{z h_{i}^{\prime \prime}(z)}{h_{i}^{\prime}(z)}\right| \leq R_{i}, \quad\left|\frac{z k_{i}^{\prime \prime}(z)}{k_{i}^{\prime}(z)}\right| \leq S_{i},
\end{gathered}
$$

$$
\sum_{i=1}^{n}\left[\left|\alpha_{i}-1\right| M_{i}+\left|\beta_{i}\right| N_{i}+\left|\delta_{i}\right|\left(R_{i}+S_{i}\right)\right] \leq \frac{(2 c+1)^{\frac{2 c+1}{2 c}}}{2},
$$

then the integral operator $\mathcal{Y}_{n}$ defined by

$$
\mathcal{Y}_{n}(z)=\int_{0}^{z} \prod_{i=1}^{n}\left[\left(\frac{f_{i}(t)}{t}\right)^{\alpha_{i}-1} \cdot\left(g_{i}(t)^{\prime}\right)^{\beta_{i}} \cdot\left(\frac{\left.h_{i}{ }^{\prime}(t)\right)}{k_{i}{ }^{\prime}(t)}\right)^{\delta_{i}}\right] d t
$$

is in the class $\mathcal{S}$.

If we consider $n=1, \delta=\gamma=\alpha$ and $\alpha_{i}-1=\beta_{i}=\gamma_{i}$ in Theorem 2.1, obtain the next corollary:

Corollary 2.7. Let $\alpha$ be complex number, Re $\alpha>0, M, N, P, Q, R, S$ real positive numbers and $f, g, h, k \in \mathcal{A}$. If

$$
\begin{gathered}
\left|\frac{z f^{\prime}(z)}{f(z)}-1\right| \leq M, \quad\left|\frac{z g^{\prime \prime}(z)}{g(z)^{\prime}}\right| \leq N, \quad\left|\frac{z h^{\prime}(z)}{h(z)}-1\right| \leq P, \\
\left|\frac{z k^{\prime}(z)}{k(z)}-1\right| \leq Q, \quad\left|\frac{z h^{\prime \prime}(z)}{h^{\prime}(z)}\right| \leq R, \quad\left|\frac{z k^{\prime \prime}(z)}{k^{\prime}(z)}\right| \leq S,
\end{gathered}
$$


for all $z \in \mathbb{U}$, and

$$
|\alpha-1|(M+N+P+Q+R+S) \leq \frac{(2 \operatorname{Re} \alpha+1)^{\frac{2 R e \alpha+1}{2 R e}}}{2},
$$

then the integral operator $\mathcal{T}$ defined by

$$
\mathcal{T}(z)=\left[\alpha \int_{0}^{z}\left(f(t) \cdot g^{\prime}(t) \cdot \frac{h(t)}{k(t)} \cdot \frac{\left.h^{\prime}(t)\right)}{k^{\prime}(t)}\right)^{\alpha-1} d t\right]^{\frac{1}{\alpha}},
$$

is in the class $\mathcal{S}$.

Theorem 2.8. Let $\gamma, \alpha_{i}, \beta_{i}, \gamma_{i}, \delta_{i}$ be complex numbers, $c=\operatorname{Re} \gamma>0$ and $f_{i}, h_{i}, k_{i} \in \mathcal{S}$, $g_{i}{ }^{\prime}, h_{i}{ }^{\prime}, k_{i}{ }^{\prime} \in \mathcal{P}$. If

$$
4 \sum_{i=1}^{n}\left|\alpha_{i}-1\right|+2 \sum_{i=1}^{n}\left|\beta_{i}\right|+8 \sum_{i=1}^{n}\left|\gamma_{i}\right|+4 \sum_{i=1}^{n}\left|\delta_{i}\right| \leq \frac{c}{2}, \quad \text { for } \quad 0<c<1
$$

or

$$
4 \sum_{i=1}^{n}\left|\alpha_{i}-1\right|+2 \sum_{i=1}^{n}\left|\beta_{i}\right|+8 \sum_{i=1}^{n}\left|\gamma_{i}\right|+4 \sum_{i=1}^{n}\left|\delta_{i}\right| \leq \frac{1}{2}, \quad \text { for } \quad c \geq 1
$$

then, for any complex numbers $\delta, R e \delta \geq c$, the integral operator $\mathcal{T}_{n}$ defined in (1.1) is in the class $\mathcal{S}$.

Proof. After the same steps as in the proof of Theorem 2.1., we get

$$
\begin{aligned}
\frac{1-|z|^{2 c}}{c}\left|\frac{z H_{n}^{\prime \prime}(z)}{H_{n}^{\prime}(z)}\right| & \leq \frac{1-|z|^{2 c}}{c} \sum_{i=1}^{n}\left[\left|\alpha_{i}-1\right|\left(\left|\frac{z f_{i}^{\prime}(z)}{f_{i}(z)}\right|+1\right)+\left|\beta_{i}\right|\left|\frac{z g_{i}^{\prime \prime}(z)}{g_{i}^{\prime}(z)}\right|\right] \\
& +\frac{1-|z|^{2 c}}{c} \sum_{i=1}^{n}\left[\left|\gamma_{i}\right|\left(\left|\frac{z h_{i}^{\prime}(z)}{h_{i}(z)}\right|+1+\left|\frac{z k_{i}^{\prime}(z)}{k_{i}(z)}\right|+1\right)\right] \\
& +\frac{1-|z|^{2 c}}{c} \sum_{i=1}^{n}\left[\left|\delta_{i}\right|\left(\left|\frac{z h_{i}^{\prime \prime}(z)}{h_{i}^{\prime}(z)}\right|+\left|\frac{z k_{i}^{\prime \prime}(z)}{k_{i}^{\prime}(z)}\right|\right)\right],
\end{aligned}
$$

for all $z \in \mathbb{U}$.

Since $f_{i}, h_{i}, k_{i} \in \mathcal{S}$ we have

$$
\left|\frac{z f_{i}^{\prime}(z)}{f_{i}(z)}\right| \leq \frac{1+|z|}{1-|z|}, \quad\left|\frac{z h_{i}^{\prime}(z)}{h_{i}(z)}\right| \leq \frac{1+|z|}{1-|z|}, \quad\left|\frac{z k_{i}^{\prime}(z)}{k_{i}(z)}\right| \leq \frac{1+|z|}{1-|z|},
$$

for all $z \in \mathbb{U}, i=\overline{1, n}$.

For $g_{i}{ }^{\prime}, h_{i}{ }^{\prime}, k_{i}{ }^{\prime} \in \mathcal{P}$ we have

$$
\left|\frac{z g_{i}^{\prime \prime}(z)}{g_{i}^{\prime}(z)}\right| \leq \frac{2|z|}{1-|z|^{2}}, \quad\left|\frac{z h_{i}^{\prime \prime}(z)}{h_{i}^{\prime}(z)}\right| \leq \frac{2|z|}{1-|z|^{2}}, \quad\left|\frac{z k_{i}^{\prime \prime}(z)}{k_{i}^{\prime}(z)}\right| \leq \frac{2|z|}{1-|z|^{2}},
$$

for all $z \in \mathbb{U}, i=\overline{1, n}$.

Using these relations we get

$$
\frac{1-|z|^{2 c}}{c}\left|\frac{z H_{n}^{\prime \prime}(z)}{H_{n}^{\prime}(z)}\right| \leq \frac{1-|z|^{2 c}}{c}\left(\frac{1+|z|}{1-|z|}+1\right) \sum_{i=1}^{n}\left|\alpha_{i}-1\right|
$$




$$
\begin{gathered}
+\frac{1-|z|^{2 c}}{c} \cdot \frac{2|z|}{1-|z|^{2}} \sum_{i=1}^{n}\left|\beta_{i}\right|+\frac{1-|z|^{2 c}}{c}\left(\frac{1+|z|}{1-|z|}+1+\frac{1+|z|}{1-|z|}+1\right) \sum_{i=1}^{n}\left|\gamma_{i}\right| \\
+\frac{1-|z|^{2 c}}{c}\left(\frac{2|z|}{1-|z|^{2}}+\frac{2|z|}{1-|z|^{2}}\right) \sum_{i=1}^{n}\left|\delta_{i}\right| \\
\leq \frac{1-|z|^{2 c}}{c} \cdot \frac{2}{1-|z|} \sum_{i=1}^{n}\left|\alpha_{i}-1\right|++\frac{1-|z|^{2 c}}{c} \cdot \frac{2|z|}{1-|z|^{2}} \sum_{i=1}^{n}\left|\beta_{i}\right| \\
+\frac{1-|z|^{2 c}}{c} \cdot \frac{4}{1-|z|} \sum_{i=1}^{n}\left|\gamma_{i}\right|+\frac{1-|z|^{2 c}}{c} \cdot \frac{4|z|}{1-|z|^{2}} \sum_{i=1}^{n}\left|\delta_{i}\right|
\end{gathered}
$$

for all $z \in \mathbb{U}$.

For $0<c<1$, we have $1-|z|^{2 c} \leq 1-|z|^{2}, z \in \mathbb{U}$ and by (2.12), we obtain

$$
\frac{1-|z|^{2 c}}{c}\left|\frac{z H_{n}^{\prime \prime}(z)}{H_{n}^{\prime}(z)}\right| \leq \frac{4}{c} \sum_{i=1}^{n}\left|\alpha_{i}-1\right|+\frac{2}{c} \sum_{i=1}^{n}\left|\beta_{i}\right|+\frac{8}{c} \sum_{i=1}^{n}\left|\gamma_{i}\right|+\frac{4}{c} \sum_{i=1}^{n}\left|\delta_{i}\right|,
$$

for all $z \in \mathbb{U}$.

From (2.10) and (2.13) we have

$$
\frac{1-|z|^{2 c}}{c}\left|\frac{z H_{n}^{\prime \prime}(z)}{H_{n}^{\prime}(z)}\right| \leq 1
$$

for all $z \in \mathbb{U}$ and $0<c<1$.

For $c \geq 1$ we have $\frac{1-|z|^{2 c}}{c} \leq 1-|z|^{2}$, for all $z \in \mathbb{U}$ and by (2.12), we obtain

$$
\frac{1-|z|^{2 c}}{c}\left|\frac{z H_{n}^{\prime \prime}(z)}{H_{n}^{\prime}(z)}\right| \leq 4 \sum_{i=1}^{n}\left|\alpha_{i}-1\right|+2 \sum_{i=1}^{n}\left|\beta_{i}\right|+8 \sum_{i=1}^{n}\left|\gamma_{i}\right|+4 \sum_{i=1}^{n}\left|\delta_{i}\right|,
$$

for all $z \in \mathbb{U}$ and $c \geq 1$.

From (2.11) and (2.15) we obtain

$$
\frac{1-|z|^{2 c}}{c}\left|\frac{z H_{n}^{\prime \prime}(z)}{H_{n}^{\prime}(z)}\right| \leq 1
$$

for all $z \in \mathbb{U}$ and $c \geq 1$.

And by (2.14), (2.16) and Lemma 1.2, it results that the integral operator $\mathcal{T}_{n}$, defined by (1.1) is in the class $\mathcal{S}$.

If we consider $\delta=1$ in Theorem 2.8, we obtain the next corollary:

Corollary 2.9. Let $\gamma, \alpha_{i}, \beta_{i}, \gamma_{i}, \delta_{i}$ be complex numbers, $0<\operatorname{Re} \gamma \leq 1$ and $f_{i}, h_{i}, k_{i} \in \mathcal{S}$, $g_{i}{ }^{\prime}, h_{i}{ }^{\prime}, k_{i}{ }^{\prime} \in \mathcal{P}$. If

$$
4 \sum_{i=1}^{n}\left|\alpha_{i}-1\right|+2 \sum_{i=1}^{n}\left|\beta_{i}\right|+8 \sum_{i=1}^{n}\left|\gamma_{i}\right|+4 \sum_{i=1}^{n}\left|\delta_{i}\right| \leq \frac{\text { Re } \gamma}{2}, \text { for } 0<c<1
$$

then the integral operator $\mathcal{F}_{n}$ defined by (2.4) belongs to the class $\mathcal{S}$.

If we consider $\delta=1$ and $\beta_{1}=\beta_{2}=\ldots=\beta_{n}=0$ in Theorem 2.8, we obtain the next corollary: 
Corollary 2.10. Let $\gamma, \alpha_{i}, \gamma_{i}, \delta_{i}$ be complex numbers, $0<$ Re $\gamma \leq 1$ and $f_{i}, h_{i}, k_{i} \in \mathcal{S}$, $h_{i}{ }^{\prime}, k_{i}{ }^{\prime} \in \mathcal{P}$. If

$$
4 \sum_{i=1}^{n}\left|\alpha_{i}-1\right|+8 \sum_{i=1}^{n}\left|\gamma_{i}\right|+4 \sum_{i=1}^{n}\left|\delta_{i}\right| \leq \frac{R e \gamma}{2}, \quad \text { for } \quad 0<c<1
$$

then the integral operator $\mathcal{X}_{n}$ defined by (2.6) belongs to the class $\mathcal{S}$.

If we consider $\delta=1$ and $\gamma_{1}=\gamma_{2}=\ldots=\gamma_{n}=0$ in Theorem 2.8, we obtain the next corollary:

Corollary 2.11. Let $\gamma, \alpha_{i}, \beta_{i}, \delta_{i}$ be complex numbers, $0<\operatorname{Re} \gamma \leq 1$ and $f_{i} \in \mathcal{S}$, $g_{i}{ }^{\prime}, h_{i}{ }^{\prime}, k_{i}{ }^{\prime} \in \mathcal{P}$. If

$$
4 \sum_{i=1}^{n}\left|\alpha_{i}-1\right|+2 \sum_{i=1}^{n}\left|\beta_{i}\right|+4 \sum_{i=1}^{n}\left|\delta_{i}\right| \leq \frac{\operatorname{Re} \gamma}{2}, \quad \text { for } \quad 0<c<1
$$

then the integral operator $\mathcal{Y}_{n}$ defined by (2.8) belongs to the class $\mathcal{S}$.

If we consider $\delta=1$ and $\alpha_{1}=\alpha_{2}=\ldots=\alpha_{n}=0$ in Theorem 2.8, we obtain the next corollary:

Corollary 2.12. Let $\gamma, \beta_{i}, \gamma_{i}, \delta_{i}$ be complex numbers, $0<\operatorname{Re} \gamma \leq 1$ and $h_{i}, k_{i} \in \mathcal{S}$, $g_{i}{ }^{\prime}, h_{i}{ }^{\prime}, k_{i}{ }^{\prime} \in \mathcal{P}$. If

$$
2 \sum_{i=1}^{n}\left|\beta_{i}\right|+8 \sum_{i=1}^{n}\left|\gamma_{i}\right|+4 \sum_{i=1}^{n}\left|\delta_{i}\right| \leq \frac{\operatorname{Re} \gamma}{2}, \quad \text { for } \quad 0<c<1
$$

then the integral operator $\mathcal{D}_{n}$ defined by (2.7) belongs to the class $\mathcal{S}$.

If we consider $\delta=1$ and $\delta_{1}=\delta_{2}=\ldots=\delta_{n}=0$ in Theorem 2.8, we obtain the next corollary:

Corollary 2.13. Let $\gamma, \alpha_{i}, \beta_{i}, \gamma_{i}$ be complex numbers, $0<$ Re $\gamma \leq 1$ and $f_{i}, h_{i}, k_{i} \in \mathcal{S}$, $g_{i}{ }^{\prime} \in \mathcal{P}$. If

$$
4 \sum_{i=1}^{n}\left|\alpha_{i}-1\right|+2 \sum_{i=1}^{n}\left|\beta_{i}\right|+8 \sum_{i=1}^{n}\left|\gamma_{i}\right| \leq \frac{\operatorname{Re} \gamma}{2}, \quad \text { for } \quad 0<c<1
$$

then the integral operator $\mathcal{S}_{n}$ defined by (2.5) belongs to the class $\mathcal{S}$.

Theorem 2.14. Let $\gamma, \delta, \alpha_{i}, \beta_{i}, \gamma_{i}, \delta_{i}$ be complex numbers, Re $\gamma>0, M_{i}, N_{i}, P_{i}$ real positive numbers and $f_{i}, g_{i}, h_{i}, k_{i} \in \mathcal{A}$. If

$$
\begin{gathered}
\left|\frac{z f_{i}^{\prime}(z)}{f_{i}(z)}-1\right| \leq M_{i}, \quad\left|\frac{z g_{i}^{\prime \prime}(z)}{g_{i}^{\prime}(z)}\right| \leq 1, \quad\left|\frac{z h_{i}^{\prime}(z)}{h_{i}(z)}-1\right| \leq N_{i}, \\
\left|\frac{z k_{i}^{\prime}(z)}{k_{i}(z)}-1\right| \leq P_{i}, \quad\left|\frac{z h_{i}^{\prime \prime}(z)}{h_{i}^{\prime}(z)}\right| \leq 1, \quad\left|\frac{z k_{i}^{\prime \prime}(z)}{k_{i}^{\prime}(z)}\right| \leq 1,
\end{gathered}
$$


for all $z \in \mathbb{U}, i=\overline{1, n}$ and

$$
\begin{aligned}
|c| & \leq 1-\frac{1}{|\delta|}\left[\left(2+M_{i}\right) \sum_{i=1}^{n}\left|\alpha_{i}-1\right|+\sum_{i=1}^{n}\left|\beta_{i}\right|\right] \\
& -\frac{1}{|\delta|}\left[\left(N_{i}+P_{i}+4\right) \sum_{i=}^{n}\left|\gamma_{i}\right|+2 \sum_{i=1}^{n}\left|\delta_{i}\right|\right]
\end{aligned}
$$

where $c \in \mathbb{C}, c \neq-1$, then the integral operator $\mathcal{T}_{n}$, defined by (1.1) is in the class $\mathcal{S}$. Proof. Also, a simple computation yields

$$
\begin{gathered}
|| c|z|^{2 \delta}+\left(1-|z|^{2 \delta}\right) \frac{z H_{n}^{\prime \prime}(z)}{\delta H_{n}^{\prime}(z)} \mid \\
\leq|c|+\frac{1}{|\delta|} \sum_{i-1}^{n}\left|\alpha_{i}-1\right|\left(\left|\frac{z f_{i}^{\prime}(z)}{f_{i}(z)}\right|+1\right)+\frac{1}{|\delta|} \sum_{i-1}^{n}\left|\beta_{i}\right|\left|\frac{z g_{i}^{\prime \prime}(z)}{g_{i}^{\prime}(z)}\right| \\
+\frac{1}{|\delta|} \sum_{i=1}^{n}\left|\gamma_{i}\right|\left[\left(\left|\frac{z h_{i}^{\prime}(z)}{h_{i}(z)}\right|+1\right)+\left(\left|\frac{z k_{i}^{\prime}(z)}{k_{i}(z)}\right|+1\right)\right] \\
+\frac{1}{|\delta|} \sum_{i=1}^{n}\left|\delta_{i}\right|\left(\left|\frac{z h_{i}^{\prime \prime}(z)}{h_{i}^{\prime}(z)}\right|+\left|\frac{z k_{i}^{\prime \prime}(z)}{k_{i}^{\prime}(z)}\right|\right)
\end{gathered}
$$

for all $z \in \mathbb{U}$.

Using these inequalities from hypothesis we have

$$
\begin{aligned}
|| c|z|^{2 \delta}+\left(1-|z|^{2 \delta}\right) \frac{z H_{n}^{\prime \prime}(z)}{\delta H_{n}^{\prime}(z)} \mid & \leq|c|+\frac{1}{|\delta|}\left[\left(2+M_{i}\right) \sum_{i=1}^{n}\left|\alpha_{i}-1\right|+\sum_{i=1}^{n}\left|\beta_{i}\right|\right] \\
& +\frac{1}{|\delta|}\left[\left(N_{i}+P_{i}+4\right) \sum_{i=}^{n}\left|\gamma_{i}\right|+2 \sum_{i=1}^{n}\left|\delta_{i}\right|\right]
\end{aligned}
$$

for all $z \in \mathbb{U}$. and hence, by inequality (2.17) we have

$$
|| c|z|^{2 \delta}+\left(1-|z|^{2 \delta}\right) \frac{z H_{n}^{\prime \prime}(z)}{\delta H_{n}^{\prime}(z)} \mid \leq 1,
$$

for all $z \in \mathbb{U}$.

Applying Lemma 1.3, we conclude that the integral operator $\mathcal{T}_{n}$, given by (1.1) is in the class $\mathcal{S}$.

If we consider $\delta=\gamma=\alpha$ and $\alpha_{i}-1=\beta_{i}=\gamma_{i}$ and $n=1$ in Theorem 2.14, we obtain the next corollary:

Corollary 2.15. Let $\alpha$ be complex number, Re $\alpha>0 \mathrm{M}, \mathrm{N}, \mathrm{P}$ real positive numbers, and $f, g, h, k \in \mathcal{A}$. If

$$
\begin{gathered}
\left|\frac{z f^{\prime}(z)}{f(z)}-1\right| \leq M, \quad\left|\frac{z g^{\prime \prime}(z)}{g^{\prime}(z)}\right| \leq 1, \quad\left|\frac{z h^{\prime}(z)}{h(z)}-1\right| \leq N, \\
\left|\frac{z k^{\prime}(z)}{k(z)}-1\right| \leq P, \quad\left|\frac{z h^{\prime \prime}(z)}{h^{\prime}(z)}\right| \leq 1, \quad\left|\frac{z k^{\prime \prime}(z)}{k^{\prime}(z)}\right| \leq 1,
\end{gathered}
$$


for all $z \in \mathbb{U}$ and

$$
|c| \leq 1-\left|\frac{\alpha-1}{\alpha}\right|\left(M_{i}+N_{i}+P_{i}+8\right), \quad c \in \mathbb{C}, \quad c \neq-1,
$$

then the integral operator $\mathcal{T}$, given by (2.9) is in the class $\mathcal{S}$.

\section{References}

[1] Aldea, C.L., Pescar, V., Univalence criteria for a general integral operator, Transilvania University of Brasov, 10(2017), no. 1, 19-30.

[2] Breaz, D., Breaz, N., Two integral operators, Studia Univ. Babeş-Bolyai Math., 47(2002), no. 3, 13-21.

[3] Breaz, D., Breaz, N., Srivastava, H.M., An extension of the univalent condition for a family of integral operators, Appl. Math. Lett., 22(2009), no. 3, 41-44.

[4] Breaz, D., Owa, S., Breaz, N., A new integral univalent operator, Acta Univ. Apulensis Math. Inform., 16(2008).

[5] Frasin, B.A., Order of convexity and univalence of general integral operator, J. Franklin Inst., 348(2011), 1013-1019.

[6] Frasin, B.A., Sufficient conditions for the univalence of an integral operator, Asia Pacific J. Math., 5(2018), no. 1, 85-91.

[7] Kim, Y.J., Merkes, E.P., On an integral of powers of a spirallike function, Kyungpook Math. J., 12(1972), 249-253.

[8] Mayer, O., The Functions Theory of the One Variable Complex, Editura Academiei, Bucureşti, Romania, 1981, 101-117.

[9] Ovesea, H., Integral operators of Bazilvic type, Bull. Math. Soc. Sci. Math. Roumanie (N.S.), 37(1993), 115-125.

[10] Ozaki, S., Nunokawa, M., The Schwarzian derivative and univalent functions, Proceedings of the American Mathematical Society, Mathematics, 33(1972), 392-394.

[11] Pascu, N.N., An improvement of Beker's univalence criterion, Proceedings of the Commemorative Session: Simion Stoïlow (Braşov, 1987), 43-48.

[12] Pascu, N.N., Pescar, V., On the integral operators of Kim-Merkes and Pfaltzgraff, Mathematica (Cluj), 32(55)(1990), no. 2, 185-192.

[13] Pescar, V., New univalence criteria for some integral operators, Studia Univ. BabesBolyai Math., 59(2014), no. 2, 185-192.

[14] Pescar, V., A new generalization of Ahlfors's and Becker's criterion of univalence, Bull. Malaysian Math. Soc., 19(1996), no. 2, 53-54.

[15] Pescar, V., Univalence criteria of certain integral operators, Acta Ciencia Indica Math, 29(2003), no. 1, 135-138.

[16] Pescar, V., On the univalence of some integral operators, General Math., Sibiu, 14(2006), no. 2, 77-84.

[17] Pescar, V., Owa, S., Univalence of certain integral operators, Int. J. Math. Math. Sci., 23(2000), 697-701.

[18] Pfaltzgraff, J.A., Univalence of the integral $f^{\prime}(z)^{\lambda}$, Bul. London Math. Soc., 7(3)(1975), 254-256. 
[19] Stanciu, L.F., The Univalence Conditions of Some Integral Operators, Hindawi Publishing Corporation, 2012.

[20] Stanciu, L.F., Breaz, D., Srivastava, H.M., Some criteria for univalence of a certain integral operator, Novi Sad J. Math., 43(2013), no. 2, 51-57.

Camelia Bărbatu

Babeş-Bolyai University,

Faculty of Mathematics and Computer Sciences,

1, Kogălniceanu Street, 400084 Cluj-Napoca, Romania

e-mail: camipode@yahoo.com

Daniel Breaz

"1 Decembrie 1918" University,

Department of Mathematics, Faculty of Exact Science and Engineering

5, Gabriel Bethlen Street,

510009 Alba-Iulia, Romania

e-mail: dbreaz@uab.ro 